\title{
Three-Center Systems for Energy Pooling: Quantum Electrodynamical Theory
}

\author{
Robert D. Jenkins ${ }^{\dagger}$ and David L. Andrews ${ }^{*, *}$ \\ School of Chemical Sciences, University of East Anglia, Norwich, NR4 7TJ, United Kingdom \\ Received: July 17, 1998; In Final Form: October 5, 1998
}

\begin{abstract}
Fundamental theory is developed for three-body resonance energy transfer in the condensed phase, involving two donors and a single acceptor. This energy pooling mechanism is responsible for recent experimental observations on trichromophore molecules and other moieties, manifest for example in the photochemistry of organo dyes and rare-earth ion doped crystals. A full quantum electrodynamical (QED) treatment of this pooling is developed and formulated with the aid of a novel diagrammatic method, which proves to have several advantages over Feynman diagram methods. Following derivation of the rate of energy pooling for an isolated group of chromophores, the electronic influences of the medium across which the energy migrates are embedded in the theory and duly discussed. Energetic constraints on the acceptor molecule are elucidated and shown to account for a variety of postulated mechanisms: the geometry of the three-center system is itself shown to exercise considerable control over the dominant mechanism. By extension, the theory is amenable to the study of more complex energy transfer arrangements, such as those observed in dendrimer chemistry and the light-harvesting photochemistry of the photosynthetic unit.
\end{abstract}

\section{Introduction}

When the absorption of light leads to electronic excitation, the associated energy is, in many cases, rapidly conveyed from the site of its initial deposition to another. This process is familiar in many areas of condensed phase photophysics, and its distance dependence is increasingly proving of value for the detailed structural analysis of proteins. ${ }^{1,2}$ When the energy transfer from donor to acceptor takes place between two chemically distinct species separated beyond wave function overlap, it has generally been considered as potentially involving two distinct mechanisms: at short distances, radiationless transfer ${ }^{3}$ with an inverse sixth power dependence on the donoracceptor separation, and at longer distances, radiative transfer identifiable with the well-known inverse square law. The comparatively recent development of a unified theory ${ }^{4}$ of resonance energy transfer, based on quantum electrodynamics (QED), has identified these two mechanisms as the long- and short-range limits of a more general, all-encompassing mechanism. With due regard to the effects of the intervening medium, ${ }^{5,6}$ the unified theory accommodates both intermolecular and interchromophore excitation transfer.

Recent studies in quite separate disciplines (vide infra) have revealed examples in the condensed phase of a fundamentally new three-center process involving the transfer of energy from two electronically preexcited donors to a single acceptor, the initial excitation of each donor having occurred through the normal absorption of a single photon. In the case of molecular species the exact vibronic level of the electronically excited donor from which energy transfers is normally a state populated by intramolecular vibrational relaxation, within the excited state manifold, prior to the interaction we here consider. In simple chemical terms the transfer itself is an ultrafast process which

†-mail: robert.jenkins@uea.ac.uk

E-mail: d.l.andrews@uea.ac.uk. Fax: +44 (0) 1603259396.

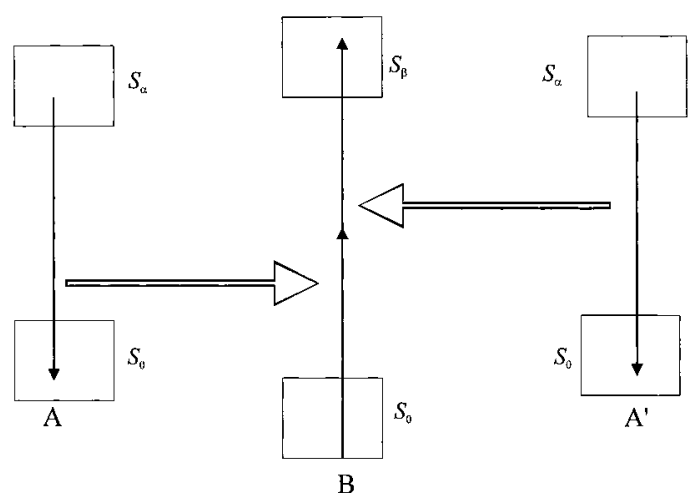

Figure 1. Modified Jablonski diagram showing the essential energetics of the three-center energy pooling process. Here $S_{0}$ represents the ground electronic state and its associated manifold; $S_{\beta}$ denotes a higher electronic excited state and its associated manifold. Vertical arrows represent transitions; horizontal arrows denote channels of excitation.

can be expressed through the equation

$$
\mathrm{A} *+\mathrm{B}+\mathrm{A} * \rightarrow \mathrm{A}+\mathrm{B} *+\mathrm{A}
$$

The above equation is to be understood as a three-center exchange of energy between essentially static moieties, not a three-body collision process. A schematic representation of the essential energetics is given in terms of a modified Jablonski diagram in Figure 1. The total energy transferred to the acceptor $\mathrm{B}$ is less than or equal to the sum of initial donor excitations, the transfer of lower energies generally associated with internal vibronic relaxation within the donor excited states.

There are several reasons for the interest in a process such as that represented above. One is that it signifies the prototypical example of potentially multicenter energy pooling processes, conceivably with several tributaries (preexcited donors) feeding a single reservoir (acceptor). Any such process affords a means of promoting the acceptor site to a state higher in energy than any individual initially excited donor. Moreover, the concerted 


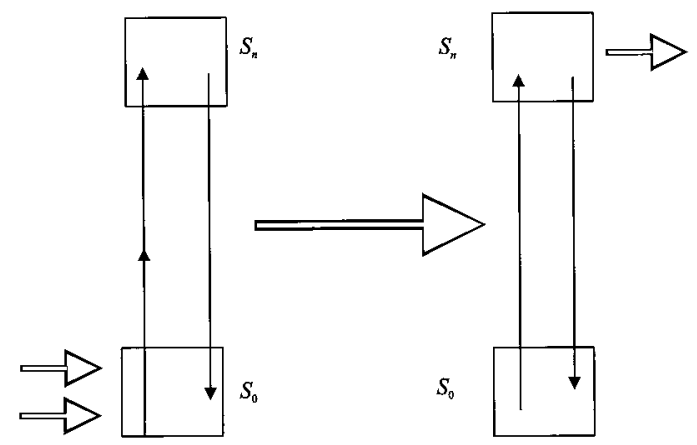

Figure 2. Modified Jablonski diagram showing the essential energetics of two-photon fluorescence resonance energy transfer.

transfer of excitation energy from two or more donors can circumvent the need, which would otherwise arise in a multistep process, for suitably placed intermediate energy levels of the acceptor.

The pooling process in particular offers all the advantages commonly associated with unimolecular two-photon absorption at $\mathrm{B}$, with the additional possibility of exploiting the intrinsic dependence on the mutual orientation of the donors and the acceptor, associated with the three-body mechanism. Also, it is only the absorber, not the site of preexcitation, that needs a two-photon absorption cross section. In other words, the initial excitation can be achieved through laser irradiation with a frequency associated with conventional single-photon absorption, giving a high population of excited state donors. Compared to the probability of two excitation photons being absorbed directly by an acceptor, there is generally a greater probability of two photons being captured by any two separate donors in its vicinity, even though both processes have an overall quadratic dependence on the pump laser intensity. ${ }^{7}$ In this sense the threebody process carries an obvious advantage over two-photon fluorescence resonant energy transfer, ${ }^{8,9}$ in which two-photon absorption at a single site is followed by energy transfer to a nearby fluorophore (see Figure 2).

Until recently, most interest in three-body donor-acceptordonor interactions has arisen in the field of rare-earth ion doped $\mathrm{Yb}^{3+}$-sensitized crystals. ${ }^{10,11}$ In such systems, atoms of the host $\mathrm{Yb}^{3+}$ lattice are excited in an absorption band away from the absorbing region of a variety of dopants, such as $\mathrm{Tm}^{3+}, \mathrm{Er}^{3+}$, and $\mathrm{Ho}^{3+}$. Here we envisage a process of energy pooling mediating the transfer of excitation energy to dopant acceptors which, in turn, emit photons at approximately twice the initial excitation energy of the lattice atoms. ${ }^{12}$ In this context, the process under consideration embraces a closed system of two initially excited lattice atoms interacting with a single atom of a dopant species. The multiatom excitation that occurs in the host lattice is considered as a pool from which any two atoms can contribute their energy. Such three-body interactions are also observed in the study of rare-earth ion doped fibers exhibiting blue emission following long-wavelength excitation. ${ }^{13}$

Three-body energy pooling has been discussed in the literature $^{11}$ as comprising both radiationless and radiative mechanisms. The former nonradiative route ${ }^{14}$ is usually thought of as proceeding not only through a cooperative process, by which the acceptor captures its energy on progression through a virtual intermediate state, but also (where the acceptor energetics allow) a stepwise excitation through two separate donor-acceptor energy transfer processes. Radiative pooling has been characterized in studies of systems sufficiently dilute in the dopant that nonradiative energy transfer is essentially negligible. ${ }^{11}$ We show here that both these mechanisms are embraced by a unified theory based on QED considerations.

The occurrence of systems that show energy migration from two identical donors to a single acceptor species is not restricted to the realm of rare-earth ion chemistry. A similar system undergoing three-body energy pooling has recently been observed in studies of simultaneous (two-photon) excitation energy transfer (SEET). ${ }^{15,16}$ SEET systems characterized thus far comprise discrete molecules of fluorescein or erythrosin as donor units, or antennae, transferring energy to an $E$-stilbene acceptor, or target. ${ }^{17}$ Acceptor and donor units are linked by thiourea units which, in themselves, inhibit both through-bond relaxation and also conjugated charge delocalization. These thiourea bridging units set the energy migration distance at $\sim 1 \AA$. As the two donor units are preexcited using laser light at a frequency off-resonant with respect to target excitation, and at intensities too low to realize direct two-photon absorption by the target, the observed intramolecular energy transfer is due to a threebody pooling interaction and is characterized via the photochemical E/Z-isomerization of the stilbene molecule. This isomerization is known to occur at energies favorable for absorption of the two donor exciting quanta. Furthermore, the sum of the exciting energies is necessarily greater than that required to initiate the isomerization, thus allowing for internal vibronic relaxation of the excited antennae, a fact experimentally recognized in SEET. ${ }^{17,18}$ SEET has the potential to open up the area of deep UV photochemistry to high-intensity longwavelength excitation.

The intramolecular pooling properties of the stilbene system cited above afford a potential springboard for other, geometrically more complex, systems where multicenter energy transfer is endowed upon a single target molecule or trap with a suitable absorbance cross section. The prototypes for these systems may be found in the area of dendrimer chemistry ${ }^{19}$ where energy hopping is observed with localized excitons in both linear chains ${ }^{20}$ and dendrimeric supermolecules. ${ }^{21,22}$ Here our theory addresses the possibility that under high levels of illumination the coexistence of more than one exciton could lead to high-energy excitation of a single trap via multibody energy pooling. Dendrimeric superstructures could yield a variety of novel many-bodied interactions, for example through their exhibition of properties analogous to light-harvesting antennae. ${ }^{23}$ This is an aspect which will be considered further in our conclusion.

\section{QED Formalism}

Three-body energy transfer involving procession of a single excitation is not unknown in the realm of QED, and the case where energy propagates along a trio of molecules has been formulated before. ${ }^{24}$ This approach can in principle be incrementally extended by molecular additions to give higher order results for energy transfer along chains of molecules, leading to a means of addressing the dynamics of energy hopping in complex systems. QED methods have also recently been applied in addressing the nonadditive three-body long-range van der Waals interaction ${ }^{25}$ by extension of methods developed in the study of the two-body potential. ${ }^{26,27}$ This method, which essentially considers each pairwise dipolar interaction among three atoms in terms of induced and spatially correlated zeropoint vacuum fluctuations "dressed" by the third, was further extended to encompass any interatomic distance beyond wave function overlap, and also to accommodate arbitrary geometrical configurations. ${ }^{28}$

For three-body energy pooling we consider not one, as in normal energy transfer, but two initial preexcitations to be 
present in the vicinity of the acceptor. Let the chemically identical donor molecules ( $\mathrm{A}$ and $\mathrm{A}^{\prime}$ ) begin in an already excited state $\left|A_{\alpha}\right\rangle$ and progress to the electronic ground state $\left|A_{0}\right\rangle$ : let the acceptor molecule (B) begin in the ground state $\left|\mathrm{B}_{0}\right\rangle$ and finish in an excited state $\left|\mathrm{B}_{\beta}\right\rangle$ via transferral of the excitation energies of the tributaries $\mathrm{A}$ and $\mathrm{A}^{\prime}$. $\mathrm{A}$ and $\mathrm{A}^{\prime}$ are considered as chemically identical molecules but necessarily distinguished for calculational purposes by features such as their distances from the acceptor B. This is important to accommodate the case of rare-earth lattice excitation where the donors need not necessarily be nearest neighbours to the acceptor. In SEET, however, the interacting donors are normally in the same molecule as the acceptor and inter-unit separation is fixed. The energetics thus satisfy the basic relation $2 E_{\alpha 0}^{\mathrm{A}}=E_{\beta 0}^{\mathrm{B}}$, where $E_{\alpha 0}^{\mathrm{A}}$ is the energy lost by a donor and $E_{\beta 0}^{\mathrm{B}}$ that gained by the acceptor. In quantum electrodynamical terms the energy migrates due to the creation and annihilation of virtual photons associated with radiation modes $(\mathbf{p}, \lambda)$ and $\left(\mathbf{p}^{\prime}, \lambda^{\prime}\right)$, the primes serving to differentiate transferral from A and $\mathrm{A}^{\prime}$. The photons are virtual-they cannot be observed, and their short propagation time which guarantees the ultrafast nature of the process implies an intrinsically high uncertainty in energy due to the timeenergy uncertainty principle. The virtual photon exchange means that molecule B must pass through a real or virtual state $\left|\mathrm{B}_{b}\right\rangle$ to get from its initial to its final state. Here we impose no restrictions on the intermediate state of $\mathrm{B}_{b}$ but its nature will be shown to play an important role in mechanistic considerations.

The full Hamiltonian for such a system, $H$, can be written as

$$
H=H_{\mathrm{mol}}^{\mathrm{A}}+H_{\mathrm{mol}}^{\mathrm{B}}+H_{\mathrm{mol}}^{\mathrm{A}^{\prime}}+H_{\mathrm{int}}^{\mathrm{A}}+H_{\mathrm{int}}^{\mathrm{B}}+H_{\mathrm{int}}^{\mathrm{A}^{\prime}}+H_{\mathrm{rad}}
$$

with $H_{\mathrm{mol}}^{\xi}$ being the molecular Hamiltonian for the molecule $\xi$, $H_{\text {rad }}$ the second-quantized radiation field Hamiltonian and $H_{\text {int }}^{\xi}$ the molecule-field coupling Hamiltonian. In the electric dipole approximation the latter operator is given by

$$
H_{\text {int }}^{\xi}=-\epsilon_{0}^{-1} \boldsymbol{\mu}(\xi) \cdot \mathbf{d}^{\perp}\left(\mathbf{R}_{\xi}\right)
$$

with $\mu(\xi)$ being the electric dipole moment operator and $\mathbf{R}_{\xi}$ the position vector of molecule $\xi$. The transverse electric displacement field operator, $\mathbf{d}^{\perp}\left(\mathbf{R}_{\xi}\right)$, can be expressed in terms of a mode expansion either in the familiar vacuum formulation or with the incorporation of media influences, as in a host or solvent "bath" ${ }^{5,29,30}$ For simplicity, eq 2.3 addresses the vacuum case (local field effects generated by bath states to be revisited at the end of this section);

$$
\begin{aligned}
\mathbf{d}^{\perp}\left(\mathbf{R}_{\xi}\right)=\sum_{\mathbf{p}, \lambda}\left(\frac{\hbar c p \epsilon_{0}}{2 V}\right)^{1 / 2} \mathrm{i}\left[\mathbf{e}^{(\lambda)}(\mathbf{p}) a^{(\lambda)}(\mathbf{p}) \mathrm{e}^{\mathrm{i} \mathbf{p} \cdot \mathbf{R}_{\xi}}-\right. \\
\left.\overline{\mathbf{e}}^{(\lambda)}(\mathbf{p}) a^{\dagger(\lambda)}(\mathbf{p}) \mathrm{e}^{-\mathrm{i} \mathbf{p} \cdot \mathbf{R}_{\xi}}\right]
\end{aligned}
$$

In expression 2.3, summation is taken over modes characterized by a wave vector $\mathbf{p}$ and polarization $\lambda$, $\mathbf{e}$ represents the electric field unit vector with $\overline{\mathbf{e}}$ being its complex conjugate, $a$ and $a^{\dagger}$ are the annihilation and creation operators, respectively, and $V$ is the quantization volume.

We can now introduce the quantum probability amplitude or matrix element, $M_{f}$, connecting the initial, $|i\rangle$, to the final, $|f\rangle$, system states via the virtual states, $|r\rangle,|s\rangle$, and $|t\rangle$, as given by
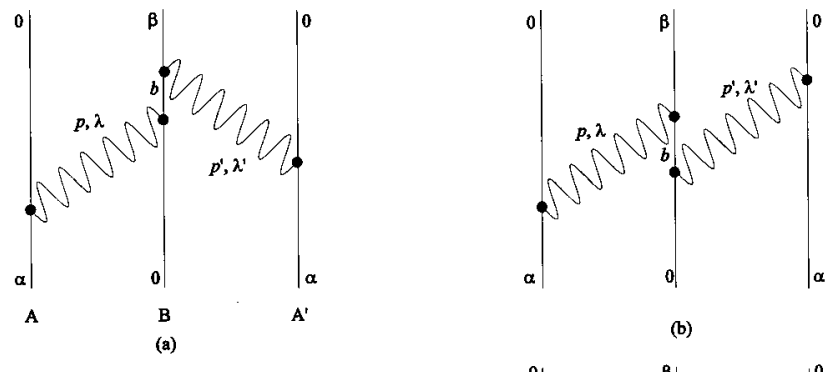

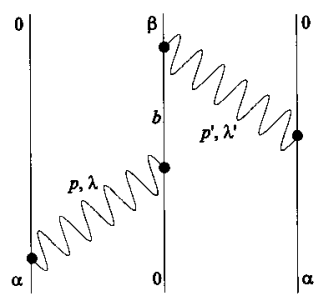

(c)

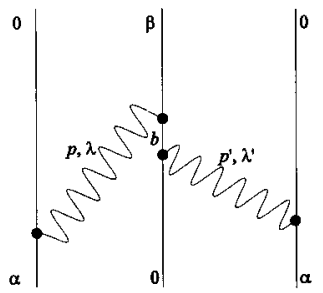

(e)

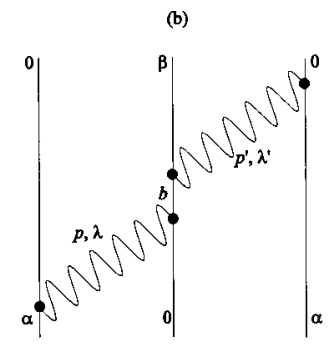

(d)

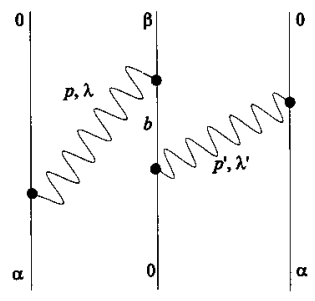

(f)
Figure 3. Six of the 24 time orderings for three-body energy pooling, the subset initialized by virtual photon creation at A. Time progresses upward. In (a), for example, the sequence is (i) creation of a virtual photon $(\mathbf{p}, \lambda)$ through decay $\alpha \rightarrow 0$ at $\mathrm{A}$, (ii) creation of virtual photon $\left(\mathbf{p}^{\prime}, \lambda^{\prime}\right)$ through decay $\alpha \rightarrow 0$ at $\mathrm{A}^{\prime}$, (iii) (p, $\lambda$ ) virtual photon annihilation at $\mathrm{B}$ accompanied by the molecular transition $0 \rightarrow b$, and (iv) $\left(\mathbf{p}^{\prime}, \lambda^{\prime}\right)$ annihilation at $\mathrm{B}$ accompanied by the transition $b \rightarrow \beta$. Eighteen further diagrams can be generated by simple temporal permutation.

the lowest order nonzero term in the time-dependent perturbation expansion, which is of fourth order since the process entails four virtual photon creation and annihilation events

$$
M_{f i}=\sum_{r, s, t} \frac{\left\langle f\left|H_{\mathrm{int}}\right| t\right\rangle\left\langle t\left|H_{\mathrm{int}}\right| s\right\rangle\left\langle s\left|H_{\mathrm{int}}\right| r\right\rangle\left\langle r\left|H_{\mathrm{int}}\right| i\right\rangle}{\left(E_{i}-E_{r}\right)\left(E_{i}-E_{s}\right)\left(E_{i}-E_{t}\right)}
$$

where $E_{n}$ is the energy of state $|n\rangle$ and the system basis states are of the form $|n\rangle=\left|\mathrm{A}_{n} ; \mathrm{A}_{n}^{\prime} ; \mathrm{B}_{n}\right\rangle\left|\operatorname{rad}_{n}\right\rangle$. The detailed form of the matrix element can be established using QED techniques ${ }^{31}$ and described in terms of a set of 24 time-ordered diagrams, six of which are shown in Figure 3. The other 18 diagrams are time-order permutations. An alternative diagrammatic technique which proves not only more compact but also calculationally more expedient in the present connection is shown in Figure 4. Here boxes represent specific combinations of the basis molecular and radiation states (and, as with time-ordered diagrams, the molecular configuration is schematic rather than geometrical). It is then possible to identify each of the 24 time orderings with routes from the initial system state on the left to the final system state on the right (see Appendix). Each intervening column of boxes represents the set of all possible virtual intermediate states, which for reference can be enumerated vertically. For example, the first set after the initial box represent the $r_{n}$ states numbered $r_{1}$ to $r_{4}$ from top to bottom.

Each individual path through the diagram begins and terminates with the same boxes, the individual states and energies of which are given below (making use of the fact that $\mathrm{A}$ and $\mathrm{A}^{\prime}$ 


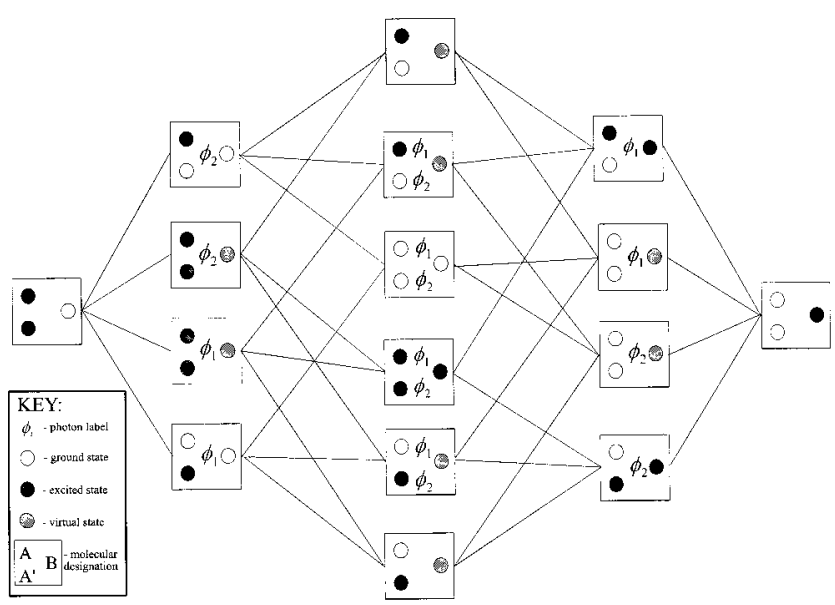

Figure 4. Box diagram for three-body energy pooling showing all 24 time orderings with the network of paths from left to right. Each box represents a combination of molecular states for the participant molecules. The labels $\phi_{1}$ and $\phi_{2}$ at the center of a box record any virtual photons present, $(\mathbf{p}, \lambda)$ and $\left(\mathbf{p}^{\prime}, \lambda^{\prime}\right)$, respectively. The six time orderings of Figure 3 correspond to paths involving box $r_{4}$.

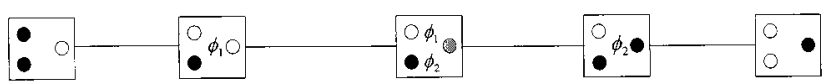

Figure 5. One of the paths through Figure 4, indicative of the specific time ordering given in Figure 3(b).

have an identical set of energy levels):

$$
\begin{array}{ll}
|i\rangle=\left|\mathrm{A}_{\alpha} ; \mathrm{A}_{\alpha}^{\prime} ; \mathrm{B}_{0}\right\rangle|0\rangle ; & E_{i}=2 E_{\alpha}^{\mathrm{A}}+E_{0}^{\mathrm{B}} \\
|f\rangle=\left|\mathrm{A}_{0} ; \mathrm{A}_{0}^{\prime} ; \mathrm{B}_{\beta}\right\rangle|0\rangle ; & E_{f}=2 E_{0}^{\mathrm{A}}+E_{\beta}^{\mathrm{B}}
\end{array}
$$

the radiation ket $|0\rangle$ being included, for completeness, to signify the vacuum state where no photons are present. Comparing, for example, Figure $3 b$ to its equivalent state sequence identified explicitly by Figure 5, we can define the path in terms of the boxes traversed:

$$
\begin{gathered}
\left.\left|r_{4}\right\rangle=\mathrm{A}_{0} ; \mathrm{A}_{\alpha}^{\prime} ; \mathrm{B}_{0}\right\rangle|1\rangle ; \quad E_{r_{4}}=E_{\alpha}^{\mathrm{A}}+E_{0}^{\mathrm{A}}+E_{0}^{\mathrm{B}}+\hbar c p \\
\left|s_{5}\right\rangle=\left|\mathrm{A}_{0} ; \mathrm{A}_{\alpha}^{\prime} ; \mathrm{B}_{b}\right\rangle\left|1 ; 1^{\prime}\right\rangle ; \\
E_{s_{5}}=E_{\alpha}^{\mathrm{A}}+E_{0}^{\mathrm{A}}+E_{b}^{\mathrm{B}}+\hbar c\left(p+p^{\prime}\right) \\
\left|t_{4}\right\rangle=\left|\mathrm{A}_{0} ; \mathrm{A}_{\alpha}^{\prime} ; \mathrm{B}_{\beta}\right\rangle\left|1^{\prime}\right\rangle ; \quad E_{t_{4}}=E_{\alpha}^{\mathrm{A}}+E_{0}^{\mathrm{A}}+E_{\beta}^{\mathrm{B}}+\hbar c p^{\prime}
\end{gathered}
$$

with the primed and unprimed numbers relating to virtual photons associated with $A$ and $A^{\prime}$ respectively, and where $\left|B_{b}\right\rangle$ is a virtual state of the acceptor, the detailed nature of which is to be discussed later. After application of (2.4), the corresponding contribution to the overall matrix element for the path, $M_{f i}^{(5)}$, is given by (2.5):

$$
\begin{gathered}
M_{f i}^{(5)}=\sum_{b, \mathbf{p}, \lambda, \mathbf{p}^{\prime}, \lambda^{\prime}}\left(\frac{\hbar c}{2 \epsilon_{0} V}\right)^{1 / 2}\left\{\mu_{i}^{0 \alpha(\mathrm{A})} \mu_{l}^{0 \alpha\left(\mathrm{A}^{\prime}\right)} \mu_{j}^{\beta b(\mathrm{~B})} \mu_{k}^{b 0(\mathrm{~B})} p \times\right. \\
\left.\bar{e}_{i}^{(\lambda)}(\mathbf{p}) e_{j}^{(\lambda)}(\mathbf{p}) \mathrm{e}^{\mathrm{i} \mathbf{p} \cdot \mathbf{R}} p^{\prime} e_{k}^{\left(\lambda^{\prime}\right)}\left(\mathbf{p}^{\prime}\right) e_{l}^{\left(\lambda^{\prime}\right)}\left(\mathbf{p}^{\prime}\right) \mathrm{e}^{-\mathrm{i} \mathbf{p}^{\prime} \cdot \mathbf{R}^{\prime}}\right\} /\left\{\left(E_{\alpha 0}^{\mathrm{A}}-\hbar c p\right) \times\right. \\
\left.\left(E_{\alpha 0}^{\mathrm{A}}-E_{b 0}^{\mathrm{B}}-\hbar c\left(p+p^{\prime}\right)\right)\left(E_{\alpha 0}^{\mathrm{A}}-E_{0 \beta}^{\mathrm{B}}-\hbar c p^{\prime}\right)\right\}
\end{gathered}
$$

introducing

$$
E_{\mathrm{xy}}^{\xi}=E_{\mathrm{x}}^{\xi}-E_{y}^{\xi}
$$

intermolecular separation vectors and $\mathbf{R}=\mathbf{R}_{\mathrm{B}}-\mathbf{R}_{\mathrm{A}}, \mathbf{R}^{\prime}=$ $\mathbf{R}_{\mathrm{B}}-\mathbf{R}_{\mathrm{A}}$,

$$
\boldsymbol{\mu}^{\mathrm{xy}(\xi)}=\left\langle\mathrm{x}\left|\boldsymbol{\mu}^{(\xi)}\right| \mathrm{y}\right\rangle
$$

and where summation over repeated Cartesian indices is implied.

Each of the separate paths has a different energy denominator associated with it. As the system is energy-conserving we can introduce the identity

$$
2 E_{\alpha 0}^{\mathrm{A}}=E_{\beta 0}^{\mathrm{B}}=2 \hbar c k
$$

defining the initial energy of each donor as $\hbar c k$. The energy $\hbar c k$ is considered fixed only in the sense that it is determined by the specific vibronic level from which donor excitation is released. Using this, and also defining the energy of any virtual state $b$ for molecule $\mathrm{B}$ as $E_{b 0}^{\mathrm{B}}=\hbar c k_{b}$ (not necessarily, or even usually, resonant with $\hbar c k$ ), the individual matrix elements can be grouped by noting that within the full set of state sequences there are four subsets that are identical in polarization features. Ignoring common factors and simplifying the notation by using $e_{n}$ for polarization components of the photon $(\mathbf{p}, \lambda)$ and $e_{n}{ }^{\prime}$ for the photon $\left(\mathbf{p}^{\prime}, \lambda^{\prime}\right)$, we first illustrate the procedure for the combination

$$
\bar{e}_{i} e_{j} \mathrm{e}^{\mathrm{i} \mathbf{p} \cdot \mathbf{R}} \bar{e}_{k}^{\prime} e_{l}^{\prime} \mathrm{e}^{-\mathrm{i} \mathbf{p}^{\prime} \cdot \mathbf{R}^{\prime}}
$$

This grouping directly links all the contributions from the pathways where one virtual photon is created at A and another at B. As represented in Figure 6, each line segment connecting successive boxes can be identified with one of the index labels $i, j, k$, or $l$. The complete set of pathways associated with this particular configuration can be simplified by adding the following set of energy denominators and dipole moment numerators:

$$
\begin{aligned}
& \bar{e}_{i} e_{j} \mathrm{e}^{\mathrm{i} \mathbf{p} \cdot \mathbf{R}} \bar{e}_{k}{ }^{\prime} e_{l}^{\prime} \mathrm{e}^{-\mathrm{i} \mathbf{p}^{\prime} \cdot \mathbf{R}^{\prime}}\left\{\frac{\mu_{k}^{\beta b(\mathrm{~B})} \mu_{j}^{b 0(\mathrm{~B})}}{(k-p)\left(k-k_{b}\right)\left(-k-p^{\prime}\right)}+\right. \\
& \frac{\mu_{j}^{\beta b(\mathrm{~B})} \mu_{k}^{b 0(\mathrm{~B})}}{(k-p)\left(k-k_{b}-p-p^{\prime}\right)\left(2 k-k_{b}-p\right)}+ \\
& \frac{\mu_{j}^{\beta b(\mathrm{~B})} \mu_{k}^{b 0(\mathrm{~B})}}{(k-p)\left(k-k_{b}-p-p^{\prime}\right)\left(-k-p^{\prime}\right)}+ \\
& \frac{\mu_{j}^{\beta b(\mathrm{~B})} \mu_{k}^{b 0(\mathrm{~B})}}{\left(-k-p^{\prime}\right)\left(k-k_{b}-p-p^{\prime}\right)\left(-k_{b}-p^{\prime}\right)}+ \\
& \frac{\mu_{j}^{\beta b(\mathrm{~B})} \mu_{k}^{b 0(\mathrm{~B})}}{\left(-k_{b}-p^{\prime}\right)\left(2 k-k_{b}-p\right)\left(k-k_{b}\right)}+ \\
& \left.\frac{\mu_{j}^{\beta b(\mathrm{~B})} \mu_{k}^{b 0(\mathrm{~B})}}{\left(-k_{b}-p^{\prime}\right)\left(k-k_{b}-p-p^{\prime}\right)\left(2 k-k_{b}-p\right)}\right\}= \\
& \frac{\mu_{j}^{\beta b(\mathrm{~B})} \mu_{k}^{b 0(\mathrm{~B})}+\mu_{j}^{\beta b(\mathrm{~B})} \mu_{k}^{b 0(\mathrm{~B})}}{\left(k-k_{b}\right)}\left\{\frac{\bar{e}_{i} e_{j} \mathrm{e}^{\mathrm{i} \mathbf{p} \cdot \mathbf{R}} \bar{e}_{k}^{\prime} e_{l}^{\prime} \mathrm{e}^{-\mathrm{i} \mathbf{p}^{\prime} \cdot \mathbf{R}^{\prime}}}{(k-p)\left(-k-p^{\prime}\right)}\right\}
\end{aligned}
$$

Implementing this method for the other three subsets and summing the results leads to a result in which it is possible to recognize a common factor identifiable as the two-photon absorption tensor $\alpha^{\beta 0(B)}$ associated with molecule B, explicitly 


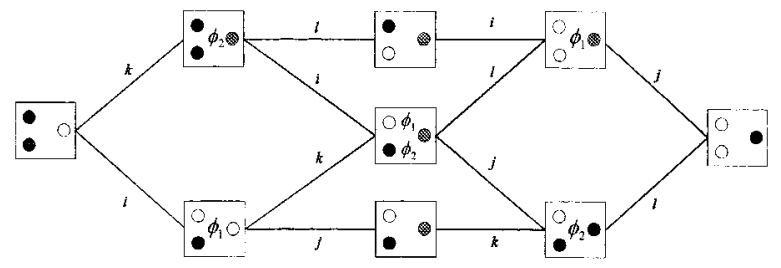

Figure 6. Full subset of Figure 4 pathways with the common radiation factor $\bar{e}_{i} e_{j} \mathrm{e}^{\mathbf{i} \mathbf{p} \cdot \mathbf{R}} \bar{e}_{k}{ }^{\prime} \boldsymbol{e}_{l}{ }^{\prime} \mathrm{e}^{-\mathrm{ip} \mathbf{p}^{\prime} \cdot \mathbf{R}^{\prime}}$. The interstate links carry index labels which signify the photons; for example, the lower left line segment labeled $i$ is associated with the creation of a virtual photon at $\mathrm{A}$, hence generating the factor.

given as

$$
\alpha_{j k}^{\beta 0(\mathrm{~B})}=\sum_{b} \frac{\mu_{j}^{\beta b(\mathrm{~B})} \mu_{k}^{b 0(\mathrm{~B})}+\mu_{k}^{\beta b(\mathrm{~B})} \mu_{j}^{b 0(\mathrm{~B})}}{\hbar c\left(k-k_{b}\right)}
$$

Expression 2.7 resembles the index-symmetric form of the $\mathbf{S}$ tensor encountered in studies of two-photon absorption and single-center two-photon fluorescence where there is an equivalence in the exciting photons. ${ }^{31}$ Using result (2.7) the total matrix element for all routes in Figure $4, M_{f i}$, can thus be written as

$$
\begin{gathered}
M_{f i}=\frac{\mu_{i}^{0 \alpha(\mathrm{A})} \mu_{l}^{0 \alpha(\mathrm{A})} \alpha_{j k}^{\beta 0(\mathrm{~B})}}{4 \epsilon_{0}^{2} V^{2}} \sum_{\mathbf{p}, \lambda \mathbf{p}^{\prime}, \lambda^{\prime}} p p^{\prime}\left\{\frac{\bar{e}_{e} e_{j} \mathrm{e}^{\mathrm{i} \mathbf{p} \cdot \mathbf{R}} \bar{e}_{k}^{\prime} e_{l}^{\prime} \mathrm{e}^{-\mathrm{i} \mathbf{p}^{\prime} \cdot \mathbf{R}^{\prime}}}{(k-p)\left(-k-p^{\prime}\right)}+\right. \\
\frac{\bar{e}_{i} e_{j} \mathrm{e}^{\mathrm{i} \cdot \mathbf{R}} \bar{e}_{k}^{\prime} \bar{e}_{l}^{\prime} \mathrm{e}^{-\mathrm{i} \mathbf{p}^{\prime} \cdot \mathbf{R}^{\prime}}}{(k-p)\left(k-p^{\prime}\right)}+\frac{\bar{e}_{i} e_{j} \mathrm{e}^{\mathrm{i} \mathbf{p} \cdot \mathbf{R}} \bar{e}_{k}^{\prime} e_{l}^{\prime} \mathrm{e}^{-\mathrm{i} \mathbf{p}^{\prime} \cdot \mathbf{R}^{\prime}}}{(k+p)\left(k+p^{\prime}\right)}+ \\
\left.\frac{e_{i} \bar{e}_{j} \mathrm{e}^{\mathrm{i} \mathbf{p} \cdot \mathbf{R}} e_{k}^{\prime} \bar{e}_{l}^{\prime} \mathrm{e}^{-\mathrm{i} \mathbf{p}^{\prime} \cdot \mathbf{R}^{\prime}}}{(-k-p)\left(k-p^{\prime}\right)}\right\}
\end{gathered}
$$

Using the identity $\sum_{\lambda} e_{i}^{(\lambda)}(\mathbf{p}) \bar{e}_{j}^{(\lambda)}(\mathbf{p})=\left(\delta_{i j}-\hat{p}_{i} \hat{p}_{j}\right)$ to carry out the polarization sums and also

$$
(1 / V) \sum_{\mathbf{p}} \Rightarrow \int \mathrm{d}^{3} \mathbf{p} /(2 \pi)^{3}
$$

to convert the $\mathbf{p}$ sums to integrals, we may then write

$$
\begin{gathered}
M_{f i}=\frac{\mu_{i}^{0 \alpha(\mathrm{A})} \mu_{l}^{0 \alpha\left(\mathrm{A}^{\prime}\right)} \alpha_{j k}^{\beta 0(\mathrm{~B})}}{256 \epsilon_{0}^{2} \pi^{6}} \int \mathrm{d}^{3} \mathbf{p} \int \mathrm{d}^{3} \mathbf{p}^{\prime} \mathrm{p}\left(\delta_{i j}-\hat{p}_{i} \hat{p}_{j}\right) p^{\prime}\left(\delta_{k l}-\right. \\
\left.\hat{p}_{k}{ }^{\prime} \hat{p}_{l}^{\prime}\right)\left\{\frac{\mathrm{e}^{\mathrm{i} \mathbf{p} \cdot \mathbf{R}} \mathrm{e}^{-\mathrm{i} \mathbf{p}^{\prime} \cdot \mathbf{R}^{\prime}}}{(k-p)\left(-k-p^{\prime}\right)}+\frac{\mathrm{e}^{\mathrm{i} \mathbf{p} \cdot \mathbf{R}} \mathrm{e}^{-\mathrm{i} \mathbf{p}^{\prime} \cdot \mathbf{R}^{\prime}}}{(k-p)\left(k-p^{\prime}\right)}+\right. \\
\left.\frac{\mathrm{e}^{\mathrm{i} \mathbf{p} \cdot \mathbf{R}} \mathrm{e}^{-\mathrm{i} \mathbf{p}^{\prime} \cdot \mathbf{R}^{\prime}}}{(k+p)\left(k+p^{\prime}\right)}+\frac{\mathrm{e}^{\mathrm{i} \mathbf{p} \cdot \mathbf{R}} \mathrm{e}^{-\mathrm{i} \mathbf{p}^{\prime} \cdot \mathbf{R}^{\prime}}}{(-k-p)\left(k-p^{\prime}\right)}\right\}
\end{gathered}
$$

With use of the identity

$$
\int p\left(\delta_{i j}-\hat{p}_{i} \hat{p}_{j}\right) \frac{\mathrm{d}^{3} \mathbf{p}}{(2 \pi)^{3}}=\left(-\nabla^{2} \delta_{i j}+\nabla_{i} \nabla_{j}\right) \int \frac{1}{p} \frac{\mathrm{d}^{3} \mathbf{p}}{(2 \pi)^{3}}
$$

with

$$
\mathrm{d}^{3} \mathbf{p}=p^{2} \mathrm{~d} p \mathrm{~d}(\cos \theta) \mathrm{d} \phi
$$

and also

$$
\int_{0}^{2 \pi} \int_{-1}^{1} \mathrm{e}^{ \pm \mathrm{i} p R \cos \theta} \mathrm{d}(\cos \theta) \mathrm{d} \phi=4 \pi \sin (p R) / p R
$$

and furthermore making use of the fact that the total integrand is odd in both $p$ and $p^{\prime}$, we can express (2.9) as follows:

$$
\begin{array}{r}
M_{f i}=\frac{\mu_{i}^{0 \alpha(\mathrm{A})} \mu_{l}^{0 \alpha\left(\mathrm{A}^{\prime}\right)} \alpha_{j k}^{\beta 0(\mathrm{~B})}}{\left(4 \epsilon_{0} \pi\right)^{2}}\left(-\nabla^{2} \delta_{i j}+\nabla_{i} \nabla_{j}\right) \frac{\mathrm{e}^{\mathrm{i} k R}}{R}\left(-\nabla^{2} \delta_{k l}+\right. \\
\left.\nabla_{k}^{\prime} \nabla^{\prime}\right) \frac{\mathrm{e}^{\mathrm{i} k R^{\prime}}}{R^{\prime}}
\end{array}
$$

Finally, using

$$
\nabla_{i} \nabla_{j} \frac{\mathrm{e}^{\mathrm{i} k R}}{R}=\frac{\mathrm{e}^{\mathrm{i} k R}}{R^{3}}\left\{(\mathrm{i} k R-1)\left(\delta_{i j}-3 \hat{R}_{i} \hat{R}_{j}\right)-k^{2} R^{2} \hat{R}_{i} \hat{R}_{j}\right\}
$$

we can write for the complete matrix element;

$$
\begin{aligned}
& M_{f i}=\frac{1}{\left(4 \pi \epsilon_{0}\right)^{2}} \mu_{i}^{0 \alpha(\mathrm{A})} \mu_{l}^{0 \alpha\left(\mathrm{A}^{\prime}\right)} \alpha_{j k}^{\beta 0(\mathrm{~B})} \frac{1}{R^{3} R^{\prime 3}}[(\mathrm{i} k R-1) \times \\
& \begin{array}{r}
\left.\left(\delta_{i j}-3 \hat{R}_{i} \hat{R}_{j}\right)-k^{2} R^{2}\left(\delta_{i j}-\hat{R}_{i} \hat{R}_{j}\right)\right]\left[\left(\mathrm{i} k R^{\prime}-1\right)\left(\delta_{k l}-3 \hat{R}_{k}^{\prime} \hat{R}_{l}^{\prime}\right)-\right. \\
\left.k^{2} R^{\prime 2}\left(\delta_{k l}-\hat{R}_{k}^{\prime} \hat{R}_{l}^{\prime}\right)\right] \mathrm{e}^{\mathrm{i} k\left(R+R^{\prime}\right)}(2.11)
\end{array}
\end{aligned}
$$

We notice embedded in eq 2.11 two second-rank, indexsymmetric, Cartesian tensors representing the retarded resonance electric dipole-electric dipole coupling, $V_{i j}(k, \mathbf{R})$ and $V_{k l}\left(k, \mathbf{R}^{\prime}\right)$, of the form ${ }^{32}$

$$
\begin{aligned}
& V_{i j}(k \cdot \mathbf{R})=\frac{\mathrm{e}^{\mathrm{i} k R}}{4 \pi \epsilon_{0} R^{3}}\left[(\mathrm{i} k R-1)\left(\delta_{i j}-3 \hat{R}_{i} \hat{R}_{j}\right)-\right. \\
&\left.k^{2} R^{2}\left(\delta_{i j}-\hat{R}_{i} \hat{R}_{j}\right)\right]
\end{aligned}
$$

In the short range, this coupling displays an $R^{-3}$ distance dependence, though the terms linear and quadratic in $k R$ increasingly modify the behavior as $R$ increases. The term "short range" in practice indicates intermolecular below $R \approx 100 \AA$ (i.e., small compared to the characteristic optical distance $k^{-1}$ ) where the radiationless limit of energy transfer dominates. As $R$ increases, retardation effects ${ }^{4,33}$ become more prominent, bringing the radiative mechanism to the fore. We are primarily interested in the short range where the coupling exerts its greatest influence, but our theory does properly accommodate retardation effects. Identification of (2.12) enables the result (2.11) to be most concisely expressed as

$$
M_{f i}=\mu_{i}^{0 \alpha(\mathrm{A})} V_{i j}(k, \mathbf{R}) \alpha_{j k}^{\beta 0(\mathrm{~B})} V_{k l}\left(k, \mathbf{R}^{\prime}\right) \mu_{l}^{0 \alpha\left(\mathrm{A}^{\prime}\right)}
$$

The rate of three-body energy pooling, $\Gamma$, can now be ascertained by substitution of the matrix element given by (2.13) into the Fermi golden rule

$$
\Gamma=(2 \pi / \hbar)\left|M_{f i}\right|^{2} \rho_{\beta}
$$

where $\rho_{\beta}$ is the appropriate density of final molecular states for the acceptor. The rate thereby acquires, in the short-range limit, an inverse sixth power dependence on both donor-acceptor distances. Usage of the Fermi rule enables us, incidentally, to compare typical rates of energy pooling with the rates of resonance energy transfer (RET) more familiar in other systems. In conventional two-body (single donor) RET, the matrix element exhibits a well-known dependence on the square of the transition dipole moments for donor and acceptor, and an $R^{-3}$ dependence on intermolecular separation. ${ }^{4}$ The matrix element for three-body energy pooling, given by (2.13), yields a dependence on the square of the two-donor transition dipole moments and a linear relation to the two-photon absorption 
tensor of the acceptor, with an $R^{-6}$ distance dependence (if $R \approx R^{\prime}$ ). Considering that a typical two-photon tensor component can realistically take a value of approximately $10^{-30} \mathrm{~m}^{3}$, then for any transfer distance of $\sim 1 \AA$ it is clear that the two types of process should offer comparable rates.

We conclude this section with a consideration of the modifications which are necessary to properly accommodate the dielectric effects of the medium across which the energy transfer occurs. In real systems the two donors and the acceptor are not isolated in a vacuum but separated by regions of space which contain other chemical species - in the case of lanthanidedoped crystals ${ }^{10,11}$ these are the ions of the host lattice, and in cases such as SEET $^{16,17}$ the intervening space is occupied by the chemically bonded structure which forms the donoracceptor linkage. To account for the electronic influence of such secondary species on the character of the energy transfer process formally requires that the theory is reformulated in terms of polaritons-virtual photons "dressed" by the secondary electric fields. The manner in which this is accomplished, detailed in several recent papers, ${ }^{5,29,30}$ is to recast the Hamiltonian of (2.1) to include contributions from all molecules beyond the interacting trio by the replacement $H_{\mathrm{rad}} \rightarrow H_{\text {bath }}$ in (2.1). This transformation is sufficient to describe the difference between the system in vacuo and under the influence of the surrounding medium. Specifically, $H_{\text {bath }}$ is given by

$$
H_{\mathrm{bath}}=H_{\mathrm{rad}}+\sum_{\xi \neq \mathrm{A}, \mathrm{A}^{\prime}, \mathrm{B}}\left[H_{\mathrm{mol}}(\xi)+H_{\mathrm{int}}(\xi)\right]
$$

with number eigenstates whose physical character is interpreted in terms of polaritons. Recasting the Hamiltonian for the interaction of $\mathrm{A}, \mathrm{A}^{\prime}$, and $\mathrm{B}$ with the bath then leads to a transformed retarded dipole-dipole coupling tensor,

$$
V_{i j}^{\text {bath }}(k, \mathbf{R})
$$

concisely expressible as

$$
V_{i j}^{\mathrm{bath}}(k, \mathbf{R})=\frac{1}{n^{2}}\left(\frac{n^{2}+2}{3}\right)^{2} V_{i j}(n k, \mathbf{R})
$$

where $n$ is the mean complex refractive index of the intervening medium at the frequencies $c k$ corresponding to the transfer energy. As a result the media-influenced matrix element is given by

$$
M_{f i}^{\mathrm{bath}}=\frac{1}{n^{4}}\left(\frac{n^{2}+2}{3}\right)^{4} \mu_{i}^{0 \alpha(\mathrm{A})} V_{i j}^{\mathrm{bath}}(n k, \mathbf{R}) \alpha_{j k}^{\beta 0(\mathrm{~B})} V_{k l}^{\mathrm{bath}}\left(n k, \mathbf{R}^{\prime}\right) \mu_{l}^{0 \alpha\left(\mathrm{A}^{\prime}\right)}
$$

If the medium immediately surrounding the twin donoracceptor system contains other excited donors, the need arises to determine the probability of finding initial excitation of the given donor pair. Outside the scope of our present work, it is a matter we shall return to elsewhere. ${ }^{34}$ The most obvious effect of the Lorentz factors combined with the other refractive features in (2.17) is to introduce a multiplicative change in the transfer rate (2.14), proving particularly significant when the energy transfer occurs in regions of high dispersion, i.e., at any optical frequency close to an absorption band. The other feature worth noting under such circumstances is the modification to the argument of the coupling tensor which, because of the imaginary component of the refractive index, leads to decay factors associated with the exponentials in (2.12). Hence both dispersive

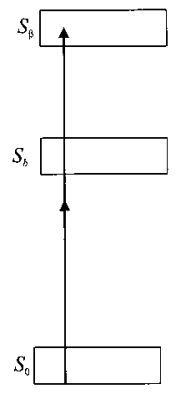

(a)

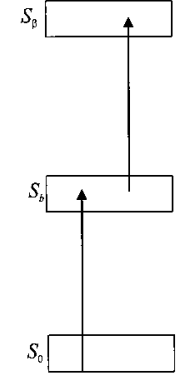

(b)

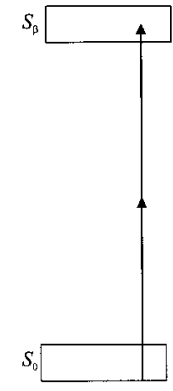

(c)
Figure 7. : Energy-level representations illustrating: (a) cooperative transfer where $b$ is considered virtual; (b) stepwise transfer where $b$ is considered as real; (c) two-level case pertaining to charge transfer where only states $\beta$ or 0 play the role of the "intermediate" state $b$. In each case the uppermost state is $\beta$.

and attenuative influences correctly feature in (2.17) and are reflected in the rate.

\section{Energetics and Charge Transfer Considerations}

The structure of the acceptor energy levels has a strong bearing on the detailed form of the results obtained in the last section, through their influence on the two-photon tensor $\alpha^{\beta 0(\mathrm{~B})}$. As given, the results directly relate to the "cooperative mechanism" illustrated in Figure 7a, in which a virtual level $b$ plays a quasi-intermediary role in the excitation of the acceptor. This case relates, for example, to the experimental results on SEET, ${ }^{17}$ for which the intermediate energy level accessed by B is deemed virtual. From eq 2.7 it is clear that, selection rules permitting, the rate increases whenever $\hbar c k$ is similar to the energy of any such state i.e., $\hbar c k_{b} \approx \hbar c k$. In the limiting case where there exists a real excited state manifold encompassing energy levels $\hbar c k$ above the ground state, as in Figure $7 \mathrm{~b}$, a stepwise excitation process becomes possible and accordingly the rate is significantly increased. Under such circumstances the finite extent of the resonance enhancement is only correctly calculable when damping factors are built into the equations, as fully described elsewhere. ${ }^{35-37}$ However, the conditions for stepwise three-body excitation will also often expedite direct two-step excitation of the acceptor, and the situation is of little theoretical interest.

Here we shall focus on another case, in which the optical response of the acceptor is dominated by transitions between just two electronic levels, the ground state and the excited state $\beta$. This quasi two-level situation will often arise when the latter is the lowest electronic excited state, as shown in Figure 7c, or where other excited states are sufficiently removed in energy to play only a minor role. Here, it transpires that a chargetransfer character to the excitation of the acceptor exercises considerable control over the rate of energy pooling, as it is also a prominent feature for two-photon absorption at a single site. ${ }^{38}$ To implement the necessary conditions we first partition the two-photon absorption tensor (2.7) into two parts, a driving term associated with charge transfer, $\alpha^{\mathrm{CT}(\mathrm{B})}$ (subject to the restriction $b=0, \beta$ alone) and a second, essentially negligible, background term $\alpha^{\mathrm{BG}(\mathrm{B}) \text {; }}$

$$
\alpha_{j k}^{\beta 0(\mathrm{~B})}=\alpha_{j k}^{C T(\mathrm{~B})}+\alpha_{j k}^{B G(\mathrm{~B})}
$$

where

$$
\alpha_{j k}^{B G(\mathrm{~B})}=\sum_{b \neq 0, \beta} \frac{\mu_{j}^{\beta b(\mathrm{~B})} \mu_{k}^{b 0(\mathrm{~B})}+\mu_{k}^{\beta b(\mathrm{~B})} \mu_{j}^{b 0(\mathrm{~B})}}{\hbar c\left(k-k_{b}\right)}
$$


and explicitly

$$
\begin{aligned}
& \alpha_{j k}^{C T(\mathrm{~B})}=\frac{\mu_{j}^{\beta \beta(\mathrm{B})} \mu_{k}^{\beta 0(\mathrm{~B})}+\mu_{k}^{\beta \beta(\mathrm{B})} \mu_{j}^{\beta 0(\mathrm{~B})}}{{ }_{1}{ }_{2} E_{\beta 0}^{\mathrm{B}}-E_{\beta 0}^{\mathrm{B}}}+ \\
& \frac{\mu_{j}^{\beta 0(\mathrm{~B})} \mu_{k}^{00(\mathrm{~B})}+\mu_{k}^{\beta 0(\mathrm{~B})} \mu_{j}^{00(\mathrm{~B})}}{1 /{ }_{2} E_{\beta 0}^{\mathrm{B}}-E_{00}^{\mathrm{B}}}
\end{aligned}
$$

Recognizing that $E_{00}^{\mathrm{B}}=0$ and denoting the static dipole moment vector difference between the ground and excited states, $\mathbf{d}^{(\mathrm{B})}$, as

$$
\mathbf{d}^{(\mathrm{B})}=\boldsymbol{\mu}^{\beta \beta(\mathrm{B})}-\boldsymbol{\mu}^{00(\mathrm{~B})}
$$

the two-photon absorption tensor approximates to

$$
\alpha_{j k}^{\beta 0(\mathrm{~B})} \approx-\frac{\mu_{j}^{\beta 0(\mathrm{~B})} d_{k}^{(\mathrm{B})}+\mu_{k}^{\beta 0(\mathrm{~B})} d_{j}^{(\mathrm{B})}}{\hbar c k}
$$

Again in passing we note that a result directly cast in terms of (3.3) can be achieved through application of a new algorithm for two-level systems. ${ }^{39,40}$ This entails effecting a transformation on the matrix element according to the prescription: leading in

$$
\mu^{\beta \beta(B)} \longmapsto\left(\mu^{\beta \beta(B)}-\mu^{00(B)}\right)=d^{(B)}, \mu^{00(B)} \mapsto 0
$$

a single step to the result given above.

The result (3.3) demonstrates that for an acceptor whose optical response is largely associated with transitions between the electronic ground state and one other electronically excited state, the rate of three-body energy pooling is linearly dependent on the magnitude of $\mathbf{d}^{(\mathrm{B})}$. This particular mechanistic channel should therefore dominate for acceptor molecules exhibiting a substantial degree of charge displacement on optical excitation.

\section{Discussion: Structure and Mechanisms}

The process of three-body energy pooling has been shown to yield potentially widespread applications across a variety of disciplines. Among these we can identify two principal classes of pooling. First, we have energy pooling systems comprising "free" species with no fixed distance between donors and acceptors. Specifically, we can identify rare-earth ion doped crystals, in which acceptor excitation can result from interactions with an undetermined number of randomly distributed excited atoms in the lattice. Second, we have a family of molecules with internal structure conducive to three-body energy pooling processes, as in SEET. Here we assume that pooling takes place within a single molecular entity, though we need not discount pooling process between these triads, such intertriad effects being governed by the first type of process. Assuming a predominance of intramolecular pooling, we may profitably investigate the effects that the fixed molecular architecture may imply.

To properly gauge the effect of molecular geometry in fixed systems, let us now assume that the two donors of interest are identical and are equidistant from the acceptor, as is commonly the case in SEET (the three entities together termed "chromophores" in the following). For further simplicity, we shall assume that effects of chromophore orientation are negligible compared to those of distance dependence, in the light of the high inverse powers with which the latter are associated. Orientational features will in any case play only a minor role in systems where the donor emission dipoles are close to parallel, a situation one can expect when the initial excitation takes place

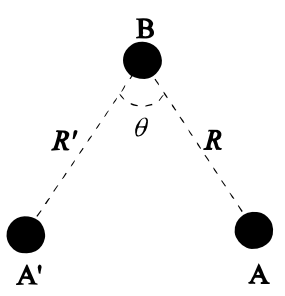

Figure 8. Three-center geometry and the mechanism-determining angle, $\theta$.

through the absorption of linearly polarized laser light. We shall also assume that the short-range form of coupling applies.

Consider the molecular geometry shown in Figure 8. In the particular case where the angle $\theta$ subtended by the donor pair at the acceptor is $60^{\circ}$, i.e., in the situation where all three interchromophore distances are equal, the two alternative mechanisms invite consideration. The energy pooling mechanism is important under such circumstances but we cannot now dismiss a further accretive mechanism. In this accretive mechanism we again consider that $\mathrm{A}$ and $\mathrm{A}^{\prime}$ are preexcited, but instead of energy transferring directly to $\mathrm{B}$ (as in normal threebody energy pooling) the excitation first migrates from one donor to the other where it "picks up" the second excitation and then moves on to B (as represented by the "box diagram" of Figure 9 in the Appendix). Again, this is a purely quantum phenomenon and the transfer of energy is nonresonant with any molecular energy levels within the second donor species. However, bearing in mind the $R^{-6}$ distance power law, it is easily shown that if $\theta$ departs from $60^{\circ}$ by as little as a few degrees, then one or the other mechanism will dominate, selection rules permitting. For example, if $\theta=62^{\circ}$, then on geometric grounds energy accretion becomes almost $20 \%$ less effective and overall we move toward the energy pooling mechanism; at $\theta=65^{\circ}$, then the accretive mechanism loses by more than $35 \%$. Taken to the extreme, when $\theta$ is $180^{\circ}$ (the case of a linear $\mathrm{ABA}^{\prime}$ configuration) there is an overwhelming bias (specifically, by a factor of 64) toward the pooling mechanism we have described. Conversely at $\theta=58^{\circ}$, accretion becomes $20 \%$ more favorable and at $55^{\circ}$, it is favored by more than $60 \%$. In general, it is evident that as $\theta$ decreases the accretive mechanism gains in significance until direct energy exchange and delocalization through wave function overlap between $\mathrm{A}$ and $\mathrm{A}^{\prime}$ becomes a consideration. The detailed theory of energy accretion is the subject of other work now in progress. ${ }^{34}$ Unless other energetic or orientational aspects of a given system have unusual significance, we can conclude that the case of equilateral displacement of the three chromophores in a twin-donor/acceptor system is one of unusual complexity, in the sense that few other geometries will necessitate consideration of more than one of the mechanisms outlined above.

One further possibility for a trigonal array arises in the context of dendrimers with the three branches "funneling" energy to a suitable acceptor core. Spectroscopic work $^{41}$ suggests that a dendrimer-like aggregate with 6-fold symmetry does not exhibit the desired exciton localization, since it is lost through ortho and parasubstitution across a phenyl linkage. The meta substitution of the $\mathrm{BA}_{3}$ aggregate acts to separate the dendrimeric chain of which it is part into regions with a distinct electronic integrity. This meta-branching acts to install a resonance decoupling of neighboring $\pi$-electron excitons. ${ }^{42}$ Thus, for a viable dendrimeric energy pooling system, the necessary meta branching suggests that accretion can play only a very minor role as $\theta$ will be close to $120^{\circ}$, leaving the mechanism detailed in the theory presented here as the major contributor. 


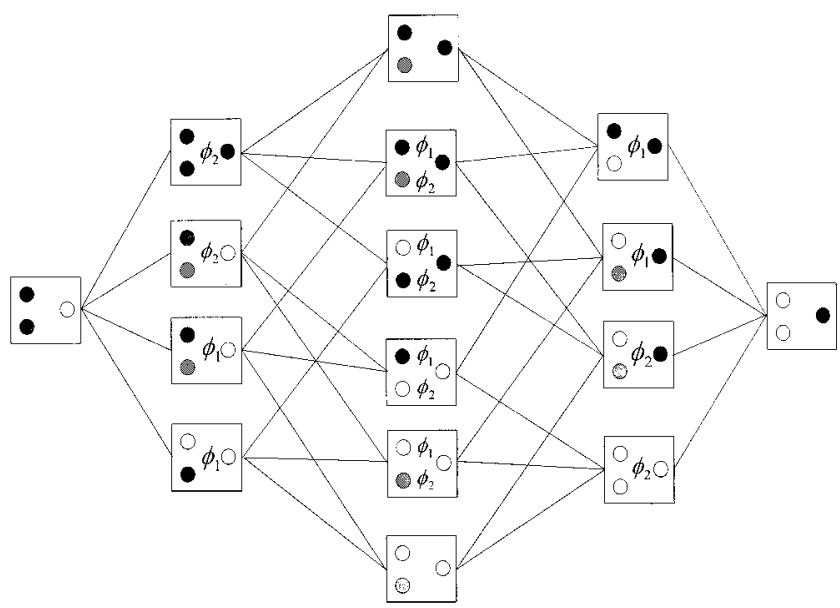

Figure 9. Box diagram showing an alternative route for the threebody process where energy migrates to the acceptor $\mathrm{B}$ from the donor species A via the other donor $\mathrm{A}^{\prime}$.

In multitributary structures the close proximity between donors will result in a degree of donor-donor coupling that might suggest the need for a further modification to the theory. In these circumstances, the initial optical excitation may lead to the establishment of Frenkel exciton states. Frenkel excitons have been shown to be important not only in photosynthetic light-harvesting complexes which comprise more than six discrete donor chromophores and an energy trap, ${ }^{43}$ but also in dendrimeric systems involving chromophores with overlapping charge distributions. ${ }^{42}$ The creation of such Frenkel states is only an issue when the number of photons initially absorbed within each superstructure is less than the number of donor species it contains, as is most often likely in such cases. The pooling of energy might then be considered in terms of excitonic tributaries. Such considerations of excitonic channelling do not, however, arise for the doubly excited two-donor case addressed in the theory we have delineated here.

The extent to which disorder restricts the coherent delocalization of excitation, in photosynthetic light-harvesting complexes $^{44}$ in particular, is an issue which remains unresolved. However, the comparatively recent structural determination of light-harvesting complexes by electron crystallography ${ }^{45,46}$ has given a new and detailed framework within which to base studies of photosynthetic energy migration ${ }^{47,48}$ with repercussions in the broader context of multiporphyrin light harvesters. ${ }^{49}$ With suitable development, application of the work presented here might then assist ongoing development of the theory for energy migration and storage within the photosynthetic unit. ${ }^{50,51}$

Acknowledgment. R.D.J. gratefully acknowledges a research studentship financed by Engineering and Physical Sciences Research Council. We also thank Philip Allcock, Luciana Dávila-Romero, and Ian D. Hands for valuable comments on this work.

\section{Appendix: Box Diagrams}

When considering the QED formulation of an optical interaction, it is usual to view the time-ordered or Feynman diagrams as representations of each contributor to the quantum amplitude of the process. Such diagrams represent successive states of the system by line segments and fundamental photonic processes by vertexes. The alternative "box diagram" representation introduced here casts the complete set of possible time orderings in a form wherein the boxes represent states and the line segments intermediary interactions. These diagrams obey the

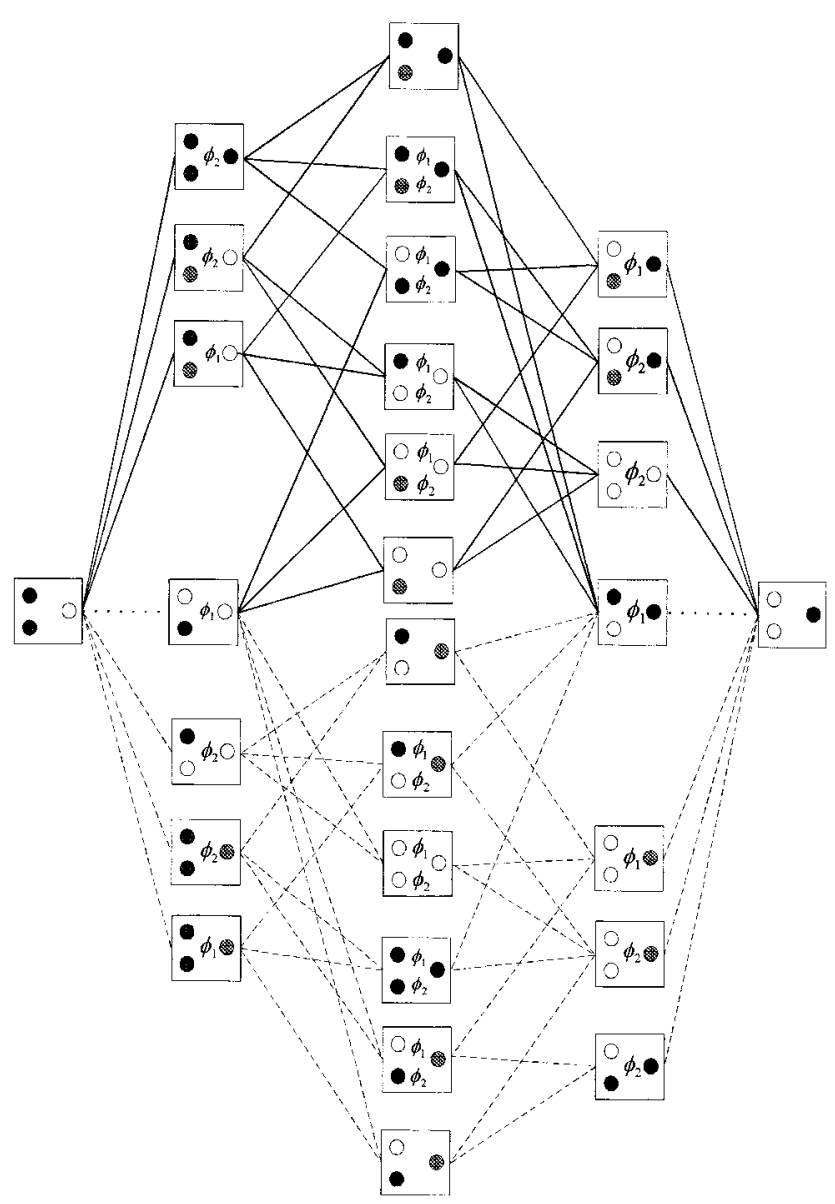

Figure 10. Full box diagram for the energy pooling process embracing both mechanistic pathways.

simple rule that only one valid change in molecular and radiation state may occur for each box-to-box progression, with respect to the system under study. The box representation of three-body energy pooling, as described in section 2, is illustrated in Figure 4. Each of the 24 complete paths from left to right represents a specific time ordering and corresponds to one time-ordered diagram.

The alternative mechanism outlined in section 4 can be represented by Figure 9. Upon inspection of Figures 9 and 4 we recognize identical boxes in the second and fourth columns, allowing us to combine the two pathways to give an overall picture of the process. The diagram in Figure 10 shows both energy pooling (dashed lines) and accretive (solid lines) aspects of the process. The equivalent paths are shown by solid lines making the inherent linkage clearly visible. All state sequences are here incorporated into the single diagram. Representation in terms of time-ordered diagrams would by contrast invoke 48 separate diagrams.

\section{References and Notes}

(1) Stryer, L. Annu. Rev. Biochem. 1978, 47, 819.

(2) Dos Remedios C. G.; Moens, P. D. J. J. Struct. Biol. 1995, 115, 175 .

(3) Förster, T. Naturwissenschaften 1946, 33, 166.

(4) Andrews, D. L. Chem. Phys. 1989, 135, 195.

(5) Juzeliūnas, G.; Andrews, D. L. Phys. Rev. B 1994, 49, 8751.

(6) Juzeliūnas, G.; Andrews, D. L. Phys. Rev. B 1994, 50, 13371

(7) Andrews, D. L. Am. J. Phys. 1985, 53, 1001.

(8) Allcock, P.; Andrews, D. L. J. Chem. Phys. 1998, 108, 3089.

(9) Allcock, P.; Andrews, D. L., in press.

(10) Seelbinder, M. B.; Wright, J. C. J. Chem. Phys. 1981, 75, 5070

(11) Chua, M.; Tanner, P. A. J. Lumin. 1996, 66 and 67, 203. 
(12) Auzel, M. F. Compt. Rend. 1966, 262B, 1016.

(13) Saïssy, A.; Dussardier, B.; Maze, G.; Monnom, G.; Wade, S. A. Opt. Fibre Technol. 1996, 2, 249.

(14) Miyakawa, T.; Dexter, D. L. Phys. Rev. B 1970, 1, 70.

(15) Bendig, J.; Bellmann, E.; Helm, S. J. Inf. Rec. Mats 1994, 21, 441. 23,66 .

A., Bendig, J. J. Inf. Recording 1996

(17) Nickoleit, M.; Uhl, A.; Bendig, J. Laser Chem. 1997, 17, 161.

(18) Sun, Y. P.; Saltiel, J. J. Phys. Chem. 1989, 93, 8310.

(19) Archut, A., Vögtle, F. Chem. Soc. Rev. 1998, 27, 233.

(20) Swallen, S. F.; Shi, Z.-Y.; Tan, W.; Xu, Z.; Moore, J. S.; Kopelman, R. J. Lumin. 1998, 76 and 77, 193

(21) Devadoss, C.; Bharathi, P.; Moore, J. S. J. Am. Chem. Soc. 1996 118,9635 .

(22) Kopelman, R.; Tan, W. Appl. Spectrosc. Rev. 1994, 29, 39

(23) Bar-Haim, A.; Klafter, J. J. Lumin. 1998, 76 and 77, 197.

(24) Craig, D. P.; Thirunamachandran, T. Chem. Phys. 1989, 135, 37. 1871.

(26) Power, E. A.; Thirunamachandran, T. Phys Rev. A 1993, 47, 2539

(27) Power, E. A.; Thirunamachandran, T. Phys Rev. A 1993, 48, 4761.

(28) Cirone M.; Passante, R. J. Phys. B: At. Mol. Opt. Phys. 1997, 30, 5579 .

(29) Juzeliūnas, G. Chem. Phys. 1995, 198, 145

(30) Juzeliūnas, G. Phys. Rev. A 1996, 53, 3543.

(31) Craig, D. P.; Thirunamachandran, T. Molecular Quantum Electrodynamics; Academic Press: New York, 1984.

(32) Power, E. A.; Thirunamachandran, T. Phys. Rev. A 1983, 28, 2671.

(33) Gomberoff, L.; Power, E. A. Proc. Phys. Soc. 1966, 88, 281.

(34) Jenkins, R. D.; Andrews, D. L. Work in progress.

(35) Barron, L. D. Molecular Light Scattering and Optical Activity; Cambridge University Press: Cambridge, UK, 1982.
(36) Butcher, P. N.; Cotter, D. The Elements of Nonlinear Optics, Cambridge University Press: Cambridge, UK, 1984.

(37) Andrews, D. L.; Naguleswaran, S.; Stedman, G. E. Phys. Rev. A 1998, 57, 4925.

(38) Meath, W. J.; Power, E. A. J. Phys. B: At. Mol. Phys. 1984, 17, 763.

(39) Kmetic, M. A.; Meath, W. J. Phys. Rev. A 1990, 41, 1556.

(40) Andrews, D. L.; Dávila-Romero, L. C.; Meath, W. J. J. Phys. B: At. Mol. Opt. Phys., in press.

(41) Kondo, K.; Yasuda, S.; Sakaguchi, T.; Miya., M. J. Chem. Soc. Chem. Commun. 1995, 1,55

(42) Tretiak, S.; Chernyak, V.; Mukamel, S. J. Phys. Chem. B 1998 $102,3310$.

(43) Zubay, G. L. Biochemistry, Wm. C. Brown, Dubuque, Iowa, 1998 (4th ed. $), 371-389$

(44) Whitmarsh, J.; Govindjee. In Encyclopedia of Applied Physics; Trigg, G. L., Ed.; VCH: New York, 1995; Vol. 13, pp 513-532.

(45) Kühlbrandt, W.; Wang, D. N. Nature 1991, 350, 130.

(46) Kühlbrandt, W.; Wang, D. N.; Fujiyoshi, Y. Nature 1994, 367, 614.

(47) Demidov, A. A.; Donovan, B.; Walker, L. A. Abst. Pap. Am. Chem. Soc. 1996, 212, 118 .

(48) Demidov, A. A.; Chernyavskaya, E. A.; Zhgirova, E. G. J. Russ. Phys. Chem. 1995, 69, 1391.

(49) Van Patten, P. G.; Shreve, A. P.; Lindsey, J. S.; Donohoe, R. J. J. Phys. Chem. B 1998, 102, 4209.

(50) Scholes, G. D.; Harcourt, R. D.; Fleming, G. R. J. Phys. Chem. B 1997, 101, 7302 .

(51) Krueger, B. P.; Scholes, G. D.; Jimenez, R.; Fleming, G. R. J. Phys. Chem. B 1998, 102, 2284. 